\title{
ASPECTOS DA TOMOGRAFIA COMPUTADORIZADA CRANIANA NA NEUROCISTICERCOSE NA INFÂNCIA
}

\author{
MARILISA M. GUERREIRO* - NUBOR O. FAOURE**-CARLOS A. M. GUERREIRO*
}

\begin{abstract}
RESUMO - Os autores relatan aspectos tomográficos da neurocisticercose na infância a propósito da análise de 27 exames em 18 pacientes, que foram distribuídos en três grupos segundo o anadru clínicu e os achados tomográficos. O grupo 1 foi caracterizado basicamtente por cpilepsias e calcıficações. $O$ grupo 2 caracterizou-se por hipertensão intracraniana e diversos aspectos tumográficos: edema, cistos e nódulos em três pacientes; hidrocefalia e calcificacóes em dois pacientes e normal em um paciente. O grupo 3 consistiu clinicamente de epilepsia e cefaléia, com vários padróes tomográficos. Os resultados são discutidos à luz da literatura disponivel.
\end{abstract}

\section{Cranial computerized tomography aspects of neurocysticercosis in infancy.}

SUMMARY - The authors present the analysis of 27 computed tonography scans (CT) of 18 children which were divided in three groups according to clinical and tomographic criteria. Group 1 was characterized mainly by epilepsy and calcifications. Group 2 wus characterized by intracranial hypertension and several tomographic aspects: edema, cysts and nodules were seen in three patients; hydrocephaly and calcifications were seen in two patients and CT was normal in one patient. Group 3 had patients with epilepsy or headache and variable tomographic patterns. The results are discussed based on the available literature.

A neurocisticercose (NC) na infância tem sido pouca registrada na literatura 1,4,6-8, pois a maioria dos trabalhos sobre cisticercose refere-se a observações em adultos. Estudos em autópsias realizados no México, Chile, Costa Rica e Peru, mostraram que freqüencia da NC na infância varia de $0,007 \%$ a $0,56 \%$ contra $1,(33 \%$ a $3,6 \%$ em adultos 7 . Em trabalho realizado em nosso meio sobre 96 pacientes com NC, mostrou-se $o$ inicio da sintomatologia na primeira década de vida em quase $30 \%$ da casuística 14. Estes dados apontam para a importância da NC na infância, apesar da menor freqüencia quando comparada à da vida adulta. A introdução da tomografia computadorizada craniana (TC) veio contribuir significativamente para a precisão diagnóstica da $N C 1,2,7,11,12$ e trouxe importante avanço no entendimento de sua lisiopatologia 6,10,12. Salienta-se ainda a importância da $\Gamma \mathrm{C}$ uma vez que o quadro clinico da NC é pleomórfico e o diagnóstico diferencial pode ser feito com muitas doenças neurológicas.

Nesta pesquisa, aspectos tomográficos da NC na infância são analisados em 27 exames que foram distribuidos em três grupos, segundo o quadro clinico e considerando os achados tomográficos.

Trabalho realizado no Departamento de Neurologia da Faculdade de Ciências Médicas (FCM) da Universidade Estadual de Campinas (UNICAMP): * Professor Assistente; ** Professor Adjunto. Este trabalho contou com o apoio financeiro de FAP - Fundo de Apoio à Pesquisa da UNICAMP, para a realização de tomografias computadorizadas cranianas. 


\section{MATERIAL E METODOS}

De agusto/1985 a dezembro/1987, 18 crianças foram consultadus no ambulatório de Neurologia InfantlI da UNICAMP como caso novo ou retorno e a partír de entăo passaram a ser acompanhadas prospectivamente em ambulatório especial, por um dos autores. Nove crianças eram do sexo feminino e 9, do masculino. Seis dessas crianças foram seguidas a partir da internação em enfermaria. O diagnóstíco de NC dado a essas crianças obedeceu aos critérios aceitos em nosso Serviço (14) - diagnóstico de possibilidade: presença de calcificações múltiplas no RX de crânio ou na TC, ou presença de calcificacāo única e liquäido cefalorraqueano (LCR) com pleucitose e imunologia especifica negativa; diagnóstico de probabilidade: LCR com pleocitose e positividade das reacões imunológicas espećfficas, ou dois ou mais cisticercos viáveis demonstrados pela TC; diagnóstico de certeza: quando a presença do cisticerco foi confirmada por cirurgia. Onze crianças receberam diagnóstico de possibilidade e 7 crianças, diagnóstico de probabilidade; năo tivemos qualquer caso de diagnóstico de certeza. Todas as crianças foram submetidas a: anamnese, exame físico, exame neurológico. eletrencefalograma, LCR, RX de crânio, TC, protoparasitológico de fezes e reacấo de Weinberg no sungue.

O proposito deste trabalho é enfocar os exames tomográficos destes pacientes. Duze crianças foram submetidas a apenas uma TC e 6 crianças a dois ou mais exames, totalizando 27 TCs analisadas.

O envolvimento do sistema nervoso central (SNC) na cisticercose pode ser classificado comı parenquimateso, ventricular, meníngeo e misto, dependendo do local comprometido (10). A TC é particularmente útil na avallação du forma parenquimatosa e pode apresentar os seguintes padrões - cisto: área central hipoatenuante, circundada ou nāo por área anelar hipercaptante devida à cápsula do cisto e edema, ao redor, de grau variável; os cistos podem ser pequenos ou grandes, únícos ou múltiplos (9); nódulo: captação homogênea de contraste de modo nodular arredondado e tende a ser menor que os cistos; sem contraste, a $T C$ pode ser normal ou mostrar pequena área de densidade diminufda; os nódulos podem ser solitários ou múltiplos e ocorrem em consequiência de morte e degeneraçăo da larva (1); greralmente medem de 1 a $2 \mathrm{~cm}$ : um terceiro padrão refere-se a áreas hipoatenuantes difusas com captação irregular de contraste e que correspondem a áreas de edema; os ventriculos são normajs ou diminuídos (9); calcificagăo: finalmente, após sua morte, o cisticerco se calcifica; as calcificaçōes sāo geralmente redondas cu ovais, medem de 2 a $6 \mathrm{~mm}$ e năo demonstram captação com contraste: ocorrem mais comumente na substáncia cinzenta ou junçăo dă substância branca com cinzenta (\&). Além destes padrões vistos na forma parenquimatoiła da NC, a TC ainda pode mostrar hidrocefalia e atrofia cerebral. Os padrôes citados foram os parâmetros en que nos baseamos para analisar os 27 exames tomográfícos.

\section{RESULTADOS}

Pudemos dividir nossos casos em três grupos aue resumem nossos achados (Tabela 1). Grupo 1 - Composto por 8 criancas que apresentavam epilepsia como manifestacão clinica predominante; as TCs evidenciaram calcificaçōes (Fig. 1A); estes casos classificam-se como forma benigna (5) ou inativa (17) da $\mathrm{NC;} 0$ tratamento medicamentoso consta apenas de

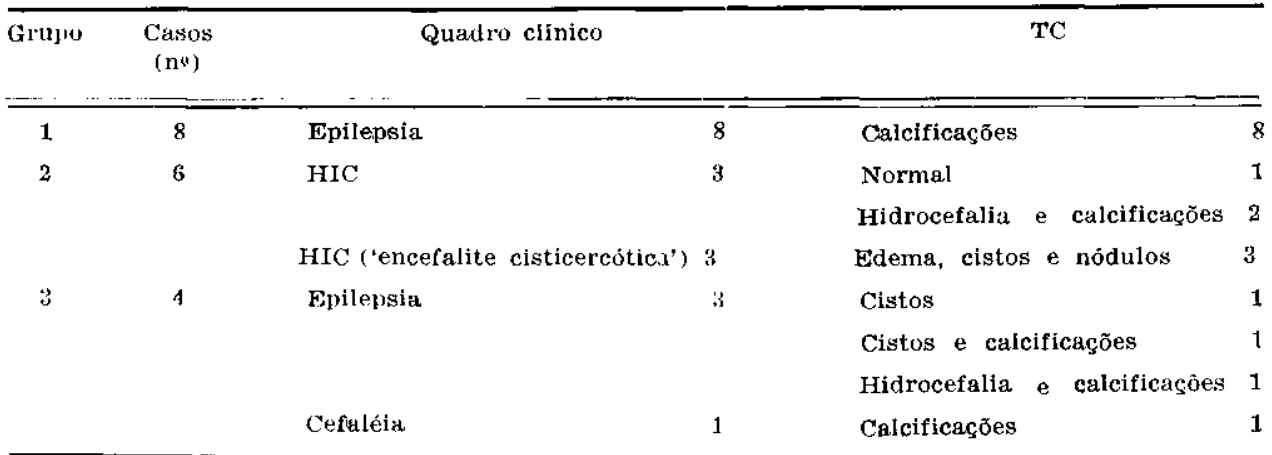

Tabela 1 - Resumo dos achados clínicos e tomograficos. HIC, hipertensão intracraniana; $T C$, tomografia computadorizada craniana. 
anticonvulsivantes. Grupo 2 - Composto por 6 crianças cujo quadro clínico predominante foi hipertensāo intracraniana (HIC); em três dessas crianças talvez o termo mais adequado fosse encefalite cisticercótica' ( 7 ), o qual expressa, além da HIC, a presença de edema cerebral e múltiplos sintomas encefaliticos; estas três crianças apresentaram TC com cistos, nódulc's e edema (Figs. 1B e 1C); duas outras crianças tinham hidrocefalia e calcificacões presentes na TC (Fig. 2A) e uma criança apresentou TC normal, que poderíamos interpretar como estário inicial da invasão aguda pelo cisticerco; esta é a forma maligna (5) ou ativa (17) da NC: duas dessas criancas foram submetidas a tratamento com praziauantel e TC poste-

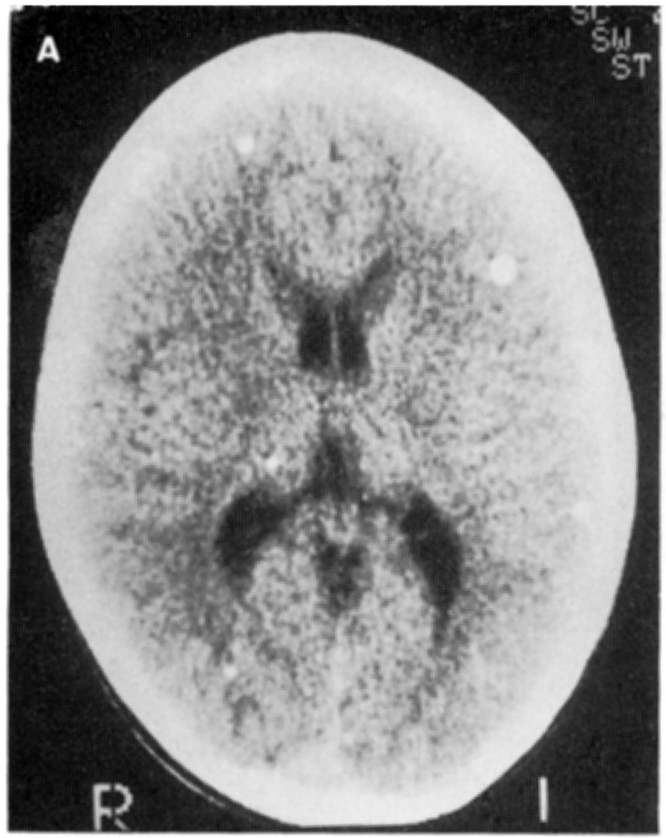

Fig. 1 - TC mostrando: em $A$, várias calcificações; em $B$ e em $C$, cistos, nódulos e edema.
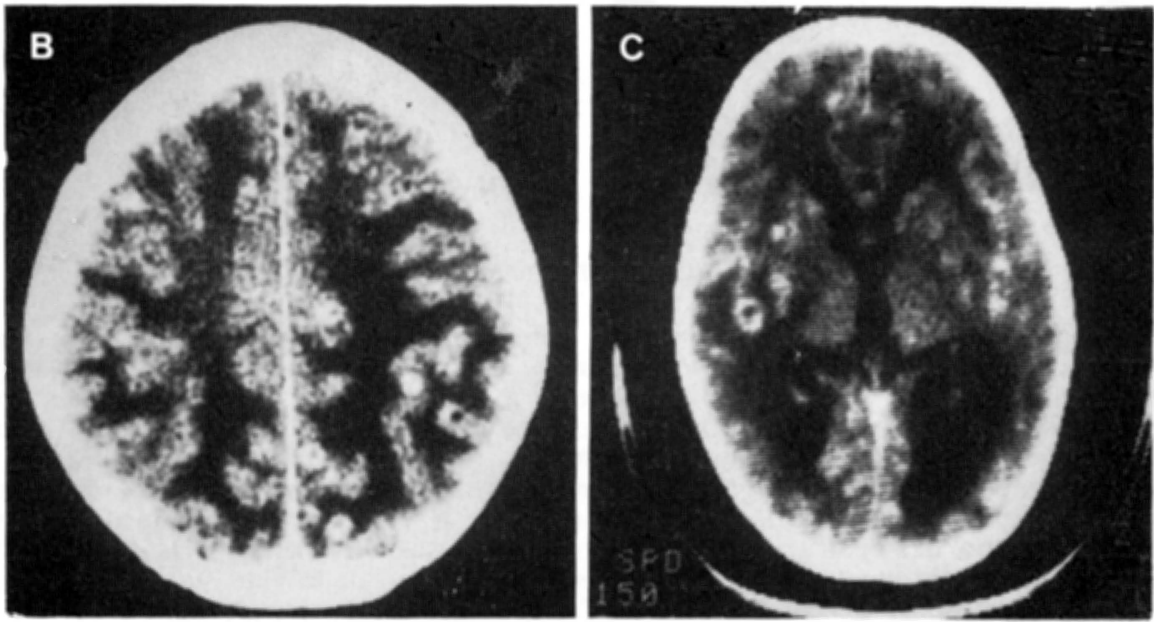

riores mostraram desaparecimento dos cistos (Fig. 2C); duas foram submetidas a derivação ventrículo-peritoneal e as outras duas foram tratadas apenas com corticóide. Grupo $3-$ Composto por 4 crianças que não se adequaram aos dois grupos anteriores; três crianças apresentavam epilensia, entretanto a TC mostrou cistos em uma delas (Fig: 2B), cistos e calcificaçōes em outra, hidrocefalia e calcificações na terceira; a quarta criança apresentava cefaléia tipo vascular e calcificaçōes na TC. 

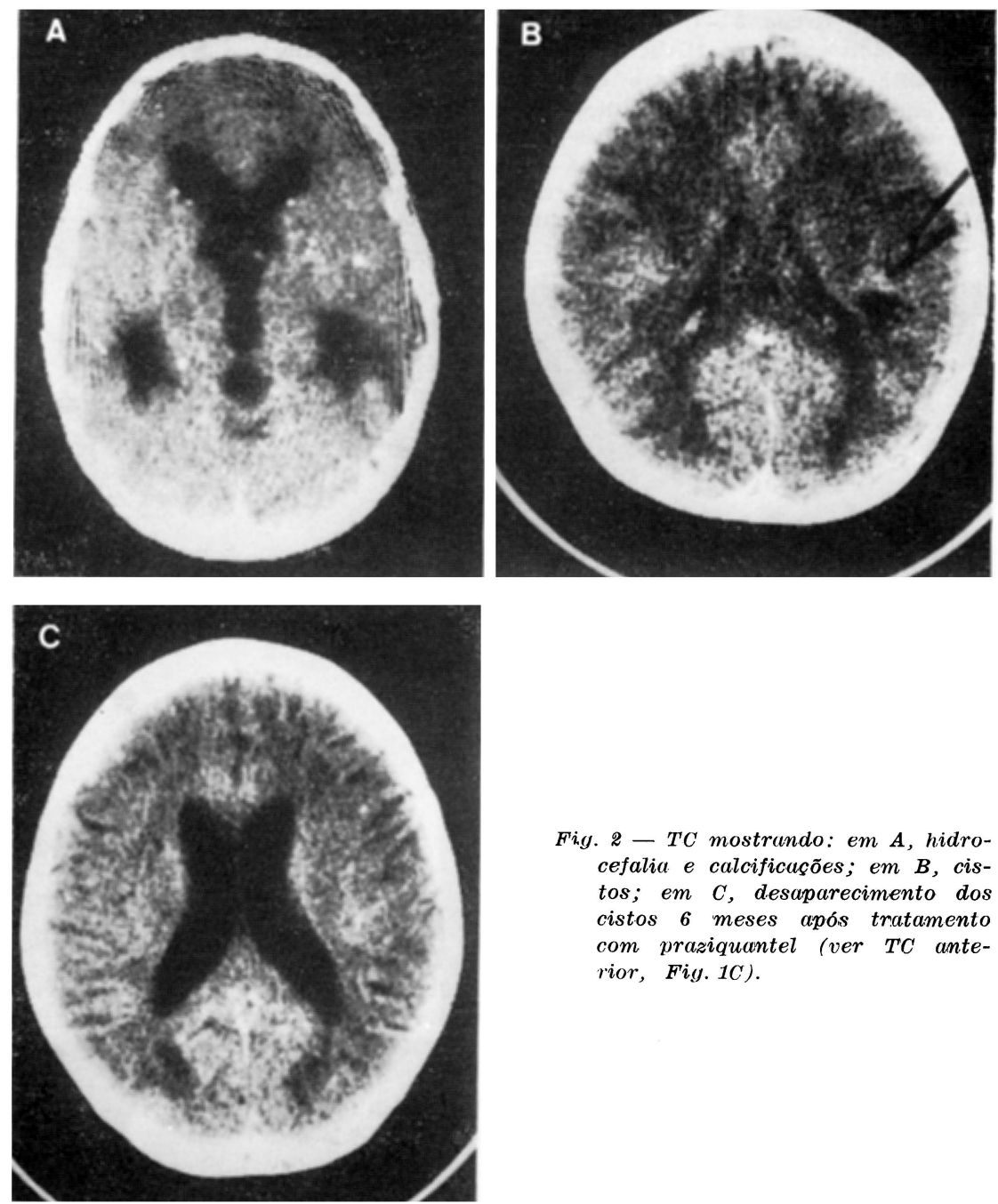

Fig. 2-TC mostrando: em A, hidrocefalia e calcificações; em $B$, cistos; em $C$, desaparecimento dos cistos 6 meses após tratamento com praziquantel (ver TC anterior, Fig. 1C).

\section{COMENTARIOS}

Os aspectos tonográficos da NC são determinados por vários fatores tais como o numero de lesóes, a localizaçăo do cisticerco no cérebro, seu tamanho, seu estágio de desenvolvimento e a resposta do hospedeiro à larva. A TC é um dos testes mais úteis para o diagnóstico da $\mathrm{NC}$ e os achados são virtualmente diagnósticos em alguns casos 12. Cisticercos em ventriculos ou meninges geralmente não são visualizados na TC e, portanto, o exame tomográfico é particularmente útil na avaliação da forma parenquimatosa da $\mathrm{NC}{ }^{13}$. Byrd e col. 2 referem que a TC é a modalidade diagnóstica mais sensivel para demonstrar as lesões parenquimatosas da doença. Dawood e col. 4 relatam que a TC foi positiva em $95 \%$ dos seus pacientes, dado este superior à reação imunológica positiva para cisticercose no LCR que foi de $87 \%$. Quagliato 14, encontrou apenas $\mathrm{um}$ exame nornal, o que significa que a TC mostrou sensibilidade diagnóstica de $99 \%$. Em nossas 8 crianças com 27 exames tomográficos, também tivemos apenas uma TC normal. Este exame foi responsável pelo diagnóstico em $50 \%$ do pacientes; na outra metade, alén da TC, tivemos também alteração do LCR sugestiva de NC.

$\mathrm{O}$ achado tomográfico mais frequente em nosso estudo foi o de cisticercos calcificacios o que concorda com várias observaçóes da literatura 9,14. As crianças com 
epilepsia e calcificações na TC foram reunidas e denominamos Grupo 1 a este conjunto. Se analisarmos o perfil etário deste grupo vamos notar que todas têm idade igual ou superior a 9 anos. O fato de não termos encontrado calcificaçōes em crianças menores que 9 anos concorda com os relatos de Byrd e col.1. Estes autores salientam que a larva morta leva de 4 a 7 anos para se calcificar, o que pode explicar porque calcificação foi mais frequentemente vista em crianças maiores. Outros autores questionam este longo tempo para aparecer calcificações. Minguetti e Ferreira 11 sugerem que lesöes agudas ou edematosas podem se calcificar num período de 23 meses após a invasão do SNC pelo parasita. Mervis e Lotz 10 observaram o aparecimento de calcificação em um paciente no periodo de um ano e salientam o fato de que calcificações foram vistas em 5 crianças com idade inferior a 5 anos, tendo sua paciente mais jovem três anos de idade. Calcificaçōes difusamente dispersas pelo parênquima cerebral provavelmente representam o estágio final da 'forma miliar' vista geralmente em crianças 1,3; das 8 crianças do grupo 1, duas apresentaram calcificações difusas. Quanto ao quadro clínico apresentado pelas crianças deste grupo nossos achados concordam com os de vários attores, os quais observaram que a maioria dos pacientes com calcificaçōes tinha convulsões $2,4,9,13,16$. Epilepsia foi a manifestação clínica mais frequente em nosso material. Alguns autores 7,8 encontraram hipertensão intracraniana (HIC) como a manifestação mais frequente. Entretanto, vale a pena ressaltar que o método de seleção dos pacientes influencia c resultado. Manreza 8, em seu estudo de 100 crianças com NC analisou pacientes internados e constatou esse fato; das nossas crianças provenientes da enfermaria, todas tiveram $\mathrm{HIC}$.

Analisando as tomografias do grupo 2, isto $\dot{e}$, de crianças que manifestaram HIC, observamos que três delas apresentaram edema cerebral, duas tinham hidrocefalia e uma teve TC normal. Este achado concorda com López-Hernandes 6,7 que notou ser o edema cerebral mais frequente que hidrocefalia como causa de HIC em crianças, ao contrário de que ocorre em adultos.

Finalmente, cabe salientar que as imagens não podem ser consideradas totalmente especificas, pois outras entidades podem ser confundidas com NC pelas TCs. Quando calcificação está presente, o diagnóstico diferencial deve ser feito com toxoplasmose e esclerose tuberosa. Nesta, as calcificaçōes são geralmente periventriculares 2, nas estruturas sub-ependimárias; as calcificações da toxoplasmose são frequentemente lineares 11. Quando cistos grandes estão presentes, o diagnóstico diferencial de NC inclui cisto aracnóideo ou porencefálico, assim como astrocitoma cístico. Cistos circundados por edema, que captam contraste revelando área semelhante a anel, devem ser diferenciados de granulomas. Lesōes múltiplas devem ser distinguidas de granulomas múltiplos 15 .

\section{REFERENCIAS}

1. Byrd SE, Locke GE, Biggers S, Percy AK - The computed tomographic appearance of cerebral cysticercosis in adults and children. Radiology 144:819, 1982.

2. Byrd SE, Percy AK, Locke GE, Smart-Abbey V - The parenchymal form of cerebral cysticercosis. Diagn Imaging 50:201, 1981.

3. Cárdenas $\mathrm{Y}$, Cárdenas J - Cysticercosis: II. Pathologic and radiologic findings. J Neurosurg 19:635, 1962.

4. Dawood AA, Moosa A - Cerebral cysticercosis in children. J Trop Pediatrics 30:136, 1984.

5. Estañol B, Corona T, Abad P - A prognostic classification of cerebral cysticercosis: therapeutic implications. J Neurol Neurosurg Psychiat 49:1131, 1986.

6. López-Hernández A - Clinical manifestations and sequential computed tomography scans of cerebral cysticercosis in childhood. Brain Dev 5:269, 1983.

7. López-Hernández A, Garaizar C - Childhood cerebral cysticercosis: clinical features and computed tomographic findings in 89 mexican children. J Canad Sci Neurol 9:401, 1982.

8. Manreza MLG - Neurocisticercose na infância: aspectos clinicos e do diagnóstico. Dissertação de Mestrado. Fac Med Unir São Paulo. São Paulo, 1981.

9. Mehringer CM, Hieshima G, Grinnell VS, Cobb SR, Zee CS, Tsai FY - Radiologio considerations in neurocysticercosis. Bull Clin Neurosci 48:21, 1983. 
10. Mervis B, Lotz JW - Computed tomography (CT) in parenchymatous cerebral cysticercosis. Clin Radiol 31:521, 1980.

11. Minguetti G, Ferreira MVC - Computed tomography in neurocysticercosis. $J$ Neurol Neurosurg Psychiat 46:936, 1983.

12. Nash TE, Neva FA - Recent advances in the diagnosis and treatment of cerebraI cysticercosis. N Engl J Med 311:1492, 1984.

13. Percy AK, Byrd SE, Locke GE - Cerebral cysticercosis. Pediatrics 66:967, 1980.

14. Quagliato EMAB - Forma epiléptica da cisticercose encefálica: análise de 96 casos. Tese. Fac Ciências Méd Univ Est Campinas. Campinas, 1987.

15. Rodriguez JC, Gutiérrez RA, Valdés OD, Dorfsman JF - The role of computed axial tomography in the diagnosis and treatment of brain inflammatory and parasitic lesions: our experience in Mexico. Neuroradiology 16:458, 1978.

16. Rodriguez-Carbajal J, Palacios E, Azar-Kia B, Churchill R - Radiology of cysticercosis of the central nervous system including computed tomography. Fadiology 125:127, 1977.

17. Sotelo J, Guerrero V, Rubio F - Neurocysticercosis: a new classification based on active and inactive forms. Arch Intern Med 145:442, 1985. 